Authors have nothing to disclose with regard to commercial support.

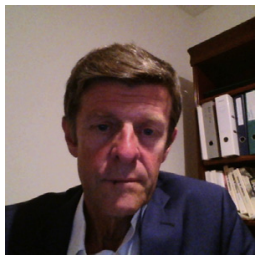

\section{CLINICAL IMPACT OF PREOPERATIVE INSPIRATORY MUSCLE TRAINING: WE NEED FURTHER STUDIES!}

\section{To the Editor:}

We read with interest the recent article by $\mathrm{Ge}$ and colleagues ${ }^{1}$ and the accompanying editorial commentary by Chou and $\mathrm{Chen}^{2}$ regarding the effectiveness of preoperative inspiratory muscle training (IMT) in improving physical fitness and reducing the incidence and importance of postoperative pulmonary complications.

Data from 13 randomized controlled trials $(\mathrm{N}=784$ patients) were collected and, after performing a meta-analysis with trial sequential analysis, the authors concluded that IMT was effective in increasing maximal inspiratory pressure by $30 \%$ and in reducing postoperative pulmonary complications by $40 \%$. The authors, however, did not examine data regarding patient compliance and withdrawal from the IMT program, factors that may contribute to the success (and failure) of this intervention and that may limit its widespread application and clinical effectiveness.

More importantly, we are concerned about 5 studies included in this meta-analysis that do not fulfill the appropriate inclusion criteria, as already pinpointed by the accompanying editorial ${ }^{2}$ :

- In the study conducted by Savci and colleagues $(\mathrm{N}=43$, cardiac surgery), ${ }^{3}$ patients underwent postoperative and preoperative IMT; moreover, maximal inspiratory pressure was not determined after IMT (before surgery).

- The Dutch team led by Dettling and colleagues ${ }^{4}$ performed a nonrandomized trial ( $\mathrm{N}=83$, esophagectomy) and allocation to IMT or usual care groups was left at the discretion of the clinicians caring for the surgical patients.

- In the study by Brocki and colleagues $(\mathrm{N}=39),{ }^{5}$ IMT was only applied postoperatively.

- Heynen and colleagues ${ }^{6}$ reported their results in a poster $(\mathrm{N}=20)$. Information regarding the conduct of the study

\footnotetext{
The Editor welcomes submissions for possible publication in the Letters to the Editor section that consist of commentary on an article published in the Journal or other relevant issues. Authors should: • Include no more than 500 words of text, three authors, and five references. • Type with double-spacing. • See http://jtcs.ctsnetjournals.org/ misc/ifora.shtml for detailed submission instructions. • Submit the letter electronically via jtcvs.editorialmanager.com. Letters commenting on an article published in the JTCVS will be considered if they are received within 6 weeks of the time the article was published. Authors of the article being commented on will be given an opportunity of offer a timely response ( 2 weeks) to the letter. Authors of letters will be notified that the letter has been received. Unpublished letters cannot be returned.
}

and full data were not available, neither on any website/ public database nor on request to the authors.

- Van Adrichem and colleagues ${ }^{7}$ compared 2 different modes of IMT, high-intensity or endurance $(\mathrm{N}=39$, esophagectomy); the IMT-endurance group cannot be considered as a control or usual care group.

If these 5 studies are excluded from the meta-analysis, the remaining 8 studies will include a total of 536 patients (instead of 784), and this would yield different results. Although we commend Ge and colleagues for their work, we would welcome an update of this meta-analysis that takes into account the concerns that have been raised hitherto.

Besides smoking cessation, correction of anemia, and improvement of nutritional status, various programs of physical training should be considered as promising nonpharmacologic interventions to secure the clinical pathway of surgical patients. ${ }^{8}$

Currently, the evidence supporting the effects of IMT on clinical study endpoints such as pneumonia or respiratory failure is weak or absent, particularly in frail patients with chronic obstructive pulmonary disease or muscular dysfunction and in those undergoing minimally invasive resection. ${ }^{8}$ Therefore, we need more unbiased randomized controlled trials to strengthen the evidence and to determine the appropriate surgical candidates and the modalities of physical training, considering patient's preference, limitations, and aptitudes in addition to the functional effectiveness of specific training programs (eg, education, IMT, aerobic fitness, muscular strength). ${ }^{2}$

Marc Licker, $M D$

John Diaper, $R A$

Department of Anesthesiology

Pharmacology \& Intensive Care

University Hospital of Geneva

Geneva, Switzerland

\section{References}

1. Ge X, Wang W, Hou L, Yang K, Fa X. Inspiratory muscle training is associated with decreased postoperative pulmonary complications: evidence from randomized trials. J Thorac Cardiovasc Surg. 2018;156:1290-300.e1295.

2. Chou $\mathrm{CH}$, Chen CY. Inspiratory muscle training for reducing postoperative pulmonary complications: a simple method that we hope is effective. J Thorac Cardiovasc Surg. 2018;156:1301-2.

3. Savci S, Degirmenci B, Saglam M, Arikan H, Inal-Ince D, Turan HN, et al. Shortterm effects of inspiratory muscle training in coronary artery bypass graft surgery: a randomized controlled trial. Scand Cardiovasc J. 2011;45:286-93.

4. Dettling DS, van der Schaaf M, Blom RL, Nollet F, Busch OR, van Berge Henegouwen MI. Feasibility and effectiveness of pre-operative inspiratory muscle training in patients undergoing oesophagectomy: a pilot study. Physiother Res Int. 2013; 18:16-26

5. Brocki BC, Andreasen JJ, Langer D, Souza DS, Westerdahl E. Postoperative inspiratory muscle training in addition to breathing exercises and early mobilization improves oxygenation in high-risk patients after lung cancer surgery: a randomized controlled trial. Eur J Cardiothoracic Surg. 2016;49:1483-91.

6. Heynen H, De Jonge C, Kerkkamp H, Willms J, Sosef M. Preconditioning in patients undergoing esophagectomy: a randomized controlled pilot study. Dis Esophagus. 2012;25:84A. 
7. van Adrichem EJ, Meulenbroek RL, Plukker JT, Groen H, van Weert E. Comparison of two preoperative inspiratory muscle training programs to prevent pulmonary complications in patients undergoing esophagectomy: a randomized controlled pilot study. Ann Surg Oncol. 2014;21:2353-60.

8. Licker M, Karenovics W, Diaper J, Fresard I, Triponez F, Ellenberger C, et al. Short-term preoperative high-intensity interval training in patients awaiting lung cancer surgery: a randomized controlled trial. J Thorac Oncol. 2017;12:323-33.

https://doi.org/10.1016/j.jtcvs.2018.09.076

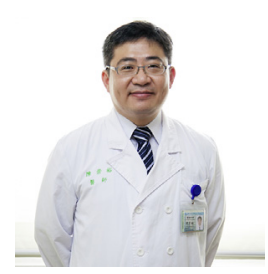

\section{INSPIRATORY MUSCLE TRAINING FOR REDUCING POSTOPERATIVE PULMONARY COMPLICATIONS: A SIMPLE METHOD BUT}

\section{SHORT OF DATA?}

\section{Reply to the Editor:}

In a letter to the Editor, Licker and Diaper ${ }^{1}$ expressed concern about the compliance and withdrawal of inspiratory muscle training (IMT) protocol and inconsistent inclusion criteria in the meta-analysis made by Ge and colleagues. ${ }^{2}$ After the exclusion of 5 studies mentioned by Licker and Diaper, ${ }^{1}$ only 536 patients (originally 784) can be analyzed in the study. That may get a different result.

A weak point of the meta-analysis is that although 13 trials were included in the study, there were only 372 patients receiving IMT and 369 patients in the control group. In addition, there were different IMT protocols in every trial. The efficacy of IMT for preventing postoperative pulmonary complications (PPCs) is also confounded by other factors that may prevent PPCs, such as smoking cessation and correcting anemia. ${ }^{3}$ These trials may indicate that IMT is one but not the only method of preventing PPCs. In the absence of confirmation by a large, randomized controlled trial, the efficacy of IMT in PPC prevention remains doubtful. We appreciate the viewpoint of Licker and Diaper, ${ }^{1}$ and we hope that Ge and colleagues will clarify the study result. The accompanying editorial commentary ${ }^{4}$ also points out that IMT is time-consuming and needs staff time. So, is IMT a simple method? Until we have standardize IMT's protocol and conducted large clinical trials with more patients, the method remains difficult.

\section{Chien-Hong Chou, MD \\ Chung-Yu Chen, MD, PhD \\ Department of Internal Medicine \\ National Taiwan University Hospital Yun-Lin Branch Yun-Lin County, Taiwan}

\section{References}

1. Licker M, Diaper J. Clinical impact of preoperative inspiratory muscle training: we need further studies! J Thorac Cardiovasc Surg. 2019;157:175-6.

2. Ge X, Wang W, Hou L, Yang K, Fa X. Inspiratory muscle training is associated with decreased postoperative pulmonary complications: evidence from randomized trials. J Thorac Cardiovasc Surg. 2018;156:1290-300.e1295.
Authors have nothing to disclose with regard to commercial support.

3. Miskovic A, Lumb AB. Postoperative pulmonary complications. Br J Anaesth. 2017:118:317-34.

4. Chou $\mathrm{CH}$, Chen CY. Inspiratory muscle training for reducing postoperative pulmonary complications: a simple method that we hope is effective. J Thorac Cardiovasc Surg. 2018;156:1301-2.

https://doi.org/10.1016/j.jtcvs.2018.10.053

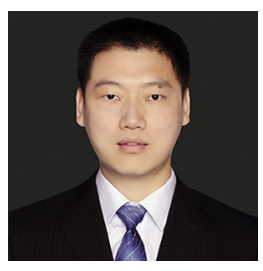

REDUCING
POSTOPERATIVE
PULMONARY
COMPLICATIONS:
STILL A LONG WAY
TO GO
Reply to the Editor:

We thank Licker and Diaper for their interest in our article. ${ }^{1}$ Postoperative pulmonary complications (PPCs) are one of the most frequent causes of postoperative morbidity and mortality. The purpose of inspiratory muscle training (IMT) is to improve inspiratory muscle strength and endurance. No powered trials, however, can confirm that IMT can decrease the incidence of PPCs. Evidence from randomized, controlled trials was insufficient to reach definitive conclusions. Our team applied the statistical method of trial sequential analysis to complete the metaanalysis and found that preoperative IMT is associated with improved maximum inspiratory pressure and decreased PPCs in patients undergoing cardiothoracic or upper abdominal surgery.

In terms of confounding factors, Licker put forward very good questions about the factors that may contribute to the success of the intervention and the inclusion criteria. We agree that these concerns may limit widespread application and clinical effectiveness. As Chou and $\mathrm{Chen}^{2}$ said in their editorial commentary, getting information from original clinical trials is a hallmark of meta-analysis, but sometimes it is not so easy. We sent email to all corresponding authors of included studies for the missing data. The study of Heynen and colleagues ${ }^{3}$ can be found in the Diseases of the Esophagus Wiley Online Library (https://onlinelibrary. wiley.com/journal/14422050), but the corresponding author's contact information did not show. One study was a nonrandomized trial, but it was a double-blind pilot study, and we evaluated the quality through the Cochrane risk of bias tool. ${ }^{4}$ The intervention in 2 studies was preoperative and postoperative IMT, which may also have had an impact on our current results. 5,6 The study by van Adrichem and colleagues ${ }^{7}$ included 2 intervention groups (a high-intensity group and an endurance group), rather 